\title{
PROPUESTA DE AUTOMATIZACIÓN DE UN PROCESO DE PRODUCCIÓN DE INÓCULO DE LEVADURA A ESCALA INDUSTRIAL PARA LA PRODUCCIÓN DE ETANOL
}

(Approach to automation of a process of yeast inoculum production on industrial scale for ethanol production)

$$
\text { *Ibeth Viviana Ordóñez Ortega, **Iber Rivera Mariño , ***Edinson Franco Mejía }
$$

*Escuela de Ingeniería Eléctrica y Electrónica, Univalle, grupo inv. GICI, ibeth.ordonez@correounivalle.edu.co

**Ingenio Providencia, Destilería, irivera@ingprovidencia.com ***Escuela de Ingeniería Eléctrica y Electrónica,

Univalle, grupo inv. GICI, edinson.franco@correounivalle.edu.co

(Recibido19 de Noviembre de 2012 y aceptado 13 de Marzo de 2013)

\begin{abstract}
Resumen:
Este artículo presenta los resultados de una investigación aplicada, orientada a automatizar la etapa de reproducción de inóculo de levadura Saccaharomyces Cerevisiae en el proceso de producción de etanol. Con base en el análisis del funcionamiento y operación del proceso a escala industrial, se identifican las variables que van a instrumentarse, los requerimientos de la instrumentación y se propone un esquema de control para el proceso.
\end{abstract}

Palabras clave: fermentación aerobia, instrumentación, control.

\section{INTRODUCCIÓN}

La etapa de reproducción de inóculo de levadura, es un proceso de fermentación aerobia. Los procesos de fermentación son ampliamente usados para obtener diferentes productos, entre ellos el etanol. En Colombia hay cinco ingenios azucareros que producen etanol a partir de la fermentación de miel B, meladura y jugo de caña. Los procesos desarrollados en este tipo de industria presentan problemas en su funcionamiento y operación ocasionados por su eje operativo, el cual es una reacción bioquímica. Esta situación reduce el rendimiento del proceso.

Concebir un esquema de automatización basado en el análisis propio del proceso a escala industrial, permite garantizar que las condiciones establecidas por la ingeniería de procesos y la microbiología, se cumplan, contribuyendo a mejorar el rendimiento. Además, el control automático de esos procesos reduce costos de operación, mejora la calidad del producto y la productividad (Thatipamala \& Hill, 1993).

Diferentes trabajos realizados en simulación, laboratorio y plantas piloto (Ashoori et al., 2008; Meleiro et al., 2009; Dovzan \& Skrjanc, 2010; Atsushi \& Aoyama, 1995; Hernández, 2007; Echeverry et al., 2003; Quintero et al., 2009), presentan novedosas técnicas de instrumentación y control para bioprocesos. Sin embargo, en escala industrial es aún difícil aplicarlas, ya que no se cuenta con herramientas comerciales lo suficientemente confiables y robustas.

\begin{abstract}
:
The results of an applied research for automation the stage of reproduction of Saccharomyces cerevisiae yeas to produce ethanol, are presented in this paper. The identification of the variables to be instrumented, the instrumentation requirements and the proposed control scheme are based on the analysis of the functioning and operation of the process.
\end{abstract}

Keywords: aerobic fermentation, instrumentation, control.

La ausencia de sensores en línea, de modelos matemáticos precisos y el comportamiento complejo y no lineal del proceso (Thatipamala\& Hill, 1993), impide concebir esquemas totalmente automáticos.

No obstante, bajo esta realidad, los procesos de fermentación en la industria deben ser operados y controlados. El control de un bioproceso a escala industrial se efectúa bajo la consigna de "proveer un ambiente cercano al óptimo para el crecimiento de los microorganismos, su reproducción y la generación de productos deseados". Esto incluye proveer una adecuada concentración de nutrientes al medio (fuente de carbono, nitrógeno, oxigeno, fosforo, sulfuros y minerales), removiendo cualquier producto metabólico toxico $\left(\mathrm{CO}_{2}\right)$ y controlando parámetros importantes de la célula como temperatura y $\mathrm{pH}$ (Alford, 2006).

Es decir, que al concebir un esquema de automatización, se deben contemplar variables que permitan garantizar unas condiciones ambientales y nutricionales adecuadas para llevar a cabo la fermentación. Bajo esta consigna se aborda el problema de la automatización del proceso de reproducción de levadura Saccaharomyces Cerevisiae, para obtener etanol.

El artículo se divide en las siguientes secciones: una descripción del funcionamiento y operación del proceso de reproducción de levadura a escala industrial, un análisis del modelo general del proceso, una reseña sobre el tipo de tecnologías empleadas para el monitoreo de las variables del proceso, y el análisis del proceso desde el punto de vista de instrumentación y control para desarrollar la propuesta de automatización. 


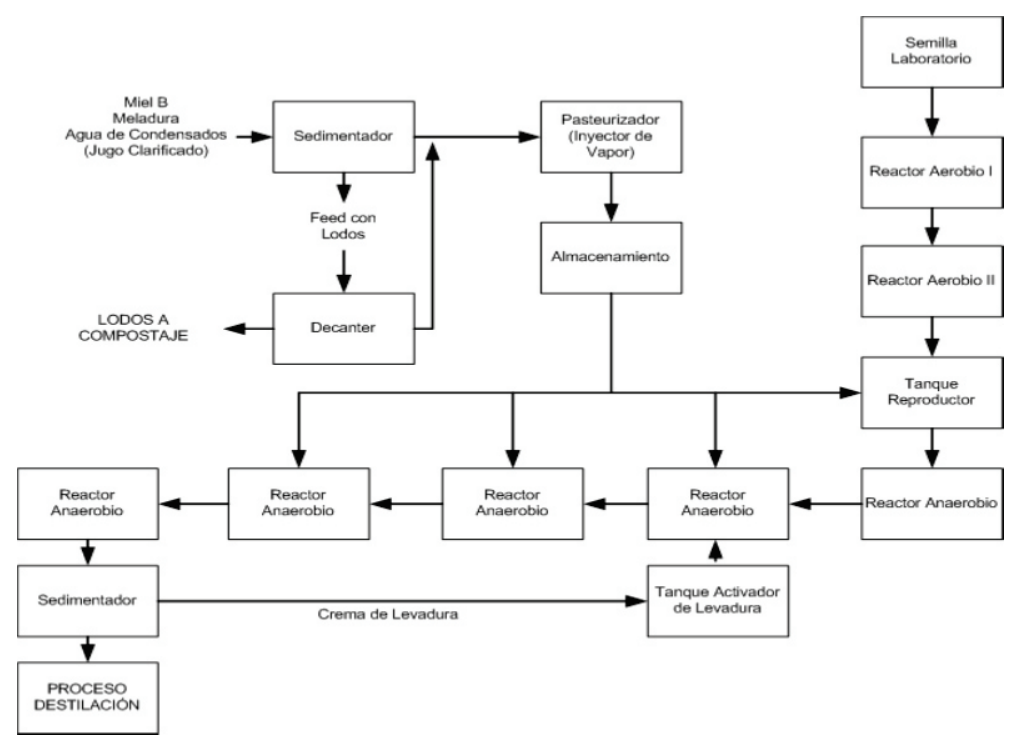

Figura 1. Esquema general del proceso de fermentación a escala industrial.

\section{DESCRIPCIÓN DEL PROCESO}

La producción de alcohol a partir de mieles y meladuras derivadas del proceso de transformación de la caña de azúcar, está conformada por varias etapas, entre la cuales se encuentran: una etapa de reacción bioquímica (fermentación), seguida por una etapa de extracción del alcohol (destilación), después una etapa de separación en la que se obtiene alcohol absoluto (deshidratación) y, por último, una etapa de tratamiento de efluentes (compostaje) (Acevedo et al., 2005).

La fermentación es un proceso en que los sustratos que componen un medio de cultivo son transformados por acción microbiana en metabolitos y biomasa (masa de células de levadura). El microorganismo va aumentando en su concentración en el transcurso del proceso, al mismo tiempo que el medio se va modificando y se forman productos nuevos como consecuencia de las actividades catabólicas y anabólicas (Merchuk, 1988).

Las destilerías cuentan con una tecnología gobernada por la operación de la levadura Sacharomyces cerevisiae en un proceso continuo, con recirculación de levadura y de vinaza. La cepa empleada tiene unas características especiales, entre las cuales están: flocular por sí misma y operar en ambientes con presencia de vinaza y etanol (Acevedo et al., 2005).

El proceso de fermentación continua (Figura 1) se inicia con la preparación y propagación de la levadura en el laboratorio (primera etapa). Posteriormente se inoculan los reactores aerobios (propagadores) en planta, garantizando un tiempo de residencia en cada uno de ellos para alcanzar las poblaciones adecuadas al proceso. A medida que cada tanque propagador cumple su tiempo de residencia, el medio va pasando al siguiente propagador hasta llegar al tanque reproductor continuo de levadura. Este tanque tiene el objetivo de maximizar la población de levadura y sostener un valor adecuado de biomasa en el resto del proceso.
Después se inyecta la levadura al tren de fermentadores anaerobios (segunda etapa), junto con la alimentación requerida para obtener un vino de alrededor $9 \%$ de concentración de alcohol. El vino pasa por un tanque sedimentador, en el cual se separa vino y crema de levadura (tercera etapa). Esta última es enviada a un tanque activador que se encarga de recuperar la levadura y enviarla nuevamente al tren de fermentación, con el fin de incrementar la eficiencia del proceso. El vino es almacenado en un tanque para ser enviado al proceso de destilación.

\subsection{Descripción del proceso de reproducción de levadura}

En ambientes industriales, las levaduras ascomicetos, como Saccharomyces cerevisiae, se reproducen vegetativamente por medio de la gemación (Lederberg \& Schaechter, 2004). Como el objetivo general de una destilería es la producción de alcohol, y este, a su vez, es un factor de estrés para la levadura, es relevante que desde el momento en que se forme el microrganismo, integre herramientas que le ayuden a lograr una mejor adaptación a medios con presencia de alcohol, y esto se logra permitiendo que se reproduzcan en medios con presencia de bajos grados alcohólicos $(\sim 2 \%)$.

Por lo anterior, en el tanque reproductor de levadura se ajustan las condiciones del proceso para que se genere alcohol, aparte del proceso fermentativo aerobio (reproducción de células de levadura) recurriendo al "efecto Crabtree", el cual es definido como la ocurrencia de la fermentación alcohólica en condiciones aeróbicas (siendo la fermentación alcohólica un proceso que se desarrolla en un ambiente anaerobio, permite que ocurra en condiciones aerobias bajo condiciones controladas).

El proceso de reproducción de levadura (ver Figura 2), está compuesto por un tanque de terminaciones cónicas, el cual cuenta con un sistema de inyección de oxigeno conformado por un sistema de filtración del aire, un sistema de flautas y dos ventiladores (blowers). Igualmente tiene acoplado un intercambiador de placas paralelas, que permite hacer el control de temperatura.
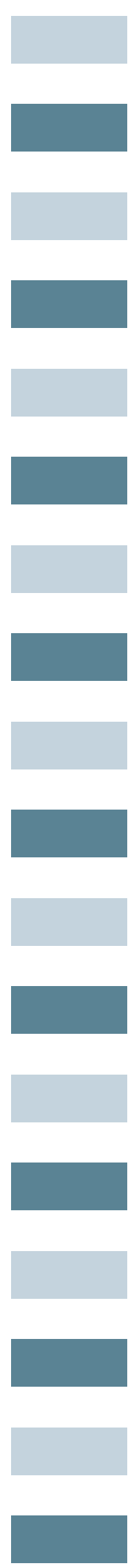


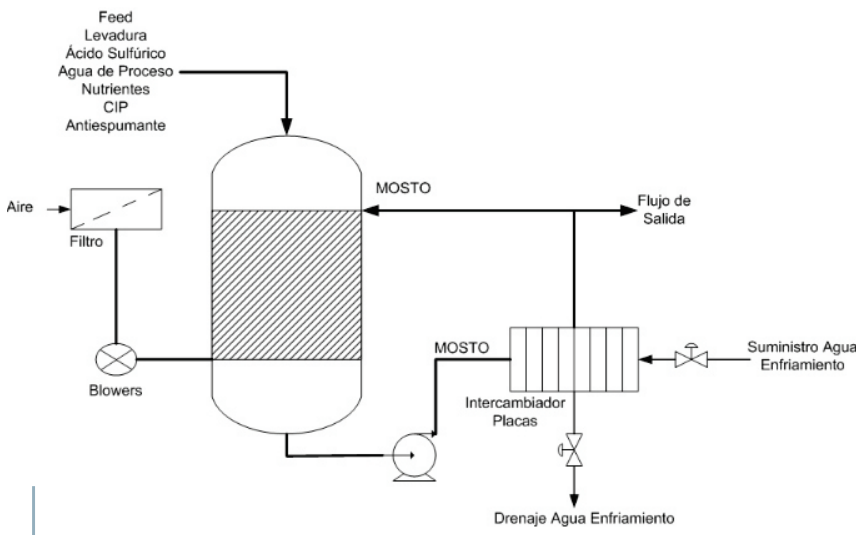

Figura 2. Descripción física del proceso de reproducción de levadura.

\section{ANÁLISIS DE CONTROL DE BIOMASA}

La concentración de biomasa es la variable central del proceso de reproducción de levaduras. Es una variable que se ve afectada por diversos parámetros (temperatura, $\mathrm{pH}$, concentración de sustrato, concentración de oxigeno, presencia de otros microorganismos, entre otros), lo cual contribuye a que sea un proceso altamente variable en el tiempo y posea una dinámica no lineal, gobernada por el metabolismo celular. $\mathrm{La}$ presencia nociva de otros microorganismos perturba significativamente cualquier proceso de estimación o monitoreo de la biomasa. Además, la ausencia de un modelo certero que permita perfilar el comportamiento de los fenómenos que se presentan en el proceso, obstaculiza aún más la tarea del control.

Considerar estrategias de control para esta variable, en procesos de reproducción de levadura que se desarrollen en destilerías del sector, es aún difícil. Para concebir un esquema de control del proceso de reproducción de levadura, el primer paso es ajustar un modelo dinámico adecuado que permita perfilar los comportamientos y fenómenos que ocurren en el proceso continuo. La literatura reporta diversos modelos para procesos de fermentación. Con base en el análisis del proceso objeto de estudio, se propone el modelo expresado en [2] (Alfoid, 2006) , [3] (Atsushi,2008), [4] (Becker ,2007) y [5] (Dochain, 1990) el cual es fundamentado por Dochain y Bastin (1990), Comerford (1992) y Mantovaneli \& Filho (1992).

La cinética de crecimiento se modela en la ecuación 1, denominado cinética de Ollson's:

Donde:

$$
\mu=\mu_{\text {MAX }}\left[\frac{S}{K_{s}+S}\right]\left[\frac{C}{K_{C}+C}\right]
$$

$\mathrm{S}=$ concentración de sustrato

$\mathrm{Ks}=$ parámetro de saturación

$\mathrm{C}=$ concentración de oxígeno disuelto

$\mu \max =$ rata máxima de crecimiento específico de la levadura $\mathrm{Kc}=$ parámetro de saturación

Balance de biomasa

$$
\frac{d X}{d t}=-\frac{F}{V} X+\mu X
$$

Donde:

$\mathrm{F}=$ flujo total de salida

$\mathrm{V}=$ volumen del medio

$\mathrm{X}=$ concentración de biomasa

Balance de Sustrato

$$
\frac{d S}{d t}=\frac{F i}{V} S i-\frac{F}{V} S-\frac{1}{Y_{X / S}} \mu X
$$

Donde:

$\mathrm{S}=$ concentración de sustrato en el medio

$\mathrm{Fi}=$ flujo de ingreso del sustrato al tanque

$\mathrm{Si}=$ concentración de entrada del sustrato (concentración inicial) $\mathrm{Yx} / \mathrm{s}=$ coeficiente de desempeño que relaciona el sustrato y la biomasa. Mide qué tanto sustrato se utilizó para producir biomasa.

Balance de etanol

Donde:

$$
\frac{d E}{d t}=-\frac{F}{V} E+\frac{Y_{F / S}}{Y_{X / S}} \mu X
$$

$\mathrm{E}=$ concentración de etanol

$\mathrm{Y} E / \mathrm{s}=$ coeficiente de desempeño que relaciona el sustrato y el etanol. Mide qué tanto sustrato se desvió para producir etanol.

Balance de Concentración de Oxígeno

$$
\frac{d C}{d t}=(Q 1-Q 2)-k 3 \mu X-D C
$$

Donde:

$\mathrm{C}=$ Concentración de oxígeno disuelto en el tanque

$\mathrm{D}=$ Rata de dilución

$\mathrm{k} 3$ = coeficiente de desempeño

$\mathrm{Q} 1$ = rata de flujo de oxigeno de entrada (por unidad de volumen)

$\mathrm{Q} 2$ = rata de flujo de oxigeno de salida (por unidad de volumen)

El modelo presenta fuertes no linealidades y parámetros que pueden variar con el tiempo, lo cual dificulta el diseño de un sistema de control.

\section{INSTRUMENTACIÓN USADA EN PROCESOS DE FERMENTACIÓN}

Los sensores más empleados en biotecnología a escala industrial, son para cuantificar el ambiente físico-químico de los bioprocesos. Variables como $\mathrm{pH}$, temperatura, presión, nivel del líquido, espuma, potencia de entrada y velocidad del impeler son monitoreadas en la mayoría de bioprocesos. Variables frecuentemente importantes, pero monitoreadas en menor cantidad, son: la viscosidad, la velocidad del fluido y las cantidades de gases. Variables como las concentraciones de sustrato, metabolitos y biomasa son poco monitoreadas por su variabilidad y complejidad. Aunque estas últimas variables pueden ser medidas mediante instrumentos que incluyen electrodos, sensores ópticos (absorbancia y fluorescencia), cromatógrafos y biosensores (Becker et al., 2007). Cabe resaltar que este tipo de tecnologías tienen restricciones que no les permite operar en todos los bioprocesos, además demandan grandes costos de adquisición y mantenimiento. 
Los proveedores de instrumentación industrial ofrecen muy pocos sensores capaces de proveer confiabilidad, mediciones directas y en línea de variables microbiológicas (p. e. concentración de biomasa) requeridas para implementar estrategias de control avanzadas (Jiang et al., 2009). Los fabricantes comerciales se enfocan en su mayoría en sensores electroquímicos para $\mathrm{pH}$ y oxígeno disuelto (Harms et al., 2002). Sin embargo existen diversas tecnologías para abordar estas mediciones, así no sean empleadas ampliamente en la industria.

Entre los desarrollos en instrumentación para bioprocesos, están los biosensores, los quimiosensores y los sensores ópticos (Becker et al., 2007). La mayoría de los sensores ópticos son basados en principios analíticos de espectroscopia. Los métodos espectroscópicos más empleados en bioprocesos son (Beutel \& Henkel, 2011): Fluorometría, Espectroscopia UV/Vis, Espectroscopia IR, Espectroscopia de Raman y Espectroscopia Terahertz.

En los diferentes bioprocesos de escala industrial, se emplean distintas tecnologías para la medición de las variables del proceso:

$p H$ : es quizás la variable más medida en procesos de fermentación. Los electrodos de vidrio son los más empleados debido a su procedimiento estandarizado de esterilización y bajos costos. También se emplean, en menor cantidad, instrumentos basados en principios ópticos como absorbancia y fluorescencia (Harms et al., 2002).

Oxígeno disuelto: el oxigeno puede ser medido en los gases de salida y en la fase líquida. Los sistemas de medición comúnmente usados son los sensores ópticos y los sensores electroquímicos convencionales (Harms et al., 2002). Los sensores electroquímicos efectúan mejores mediciones en altas concentraciones de oxígeno, mientras que los sensores ópticos son más sensibles a concentraciones más bajas (Harms et al., 2002). El sensado óptico de oxígeno es un método no reactivo, mientras que el electrodo electroquímico disminuye la concentración de oxígeno. Sin embargo, el sensor óptico tiene limitaciones en su estabilidad (Beutel \& Henkel, 2011).

Temperatura: los sensores comúnmente usados son basados en la medición de la resistencia eléctrica de un termistor de platino (RTD). Aunque en menor proporción de uso, la medición de la temperatura se encuentra disponible con métodos electroquímicos (Beutel \& Henkel, 2011).

Concentración de biomasa y población celular: esta variable puede ser medida indirectamente por medios eléctricos (capacitancia o permitividad) o medio ópticos (espectroscopia, absorbancia). La absorbancia o densidad óptica es una medida con mayores bondades que la dispersión. Para el diseño de estos sensores, se debe escoger entre ofrecer mayor rango o mejor precisión. Estos sensores son vulnerables a interferencias de partículas y burbujas de gas. La masa celular es también frecuentemente estimada por medio de sensores software (Harms et al., 2002). No obstante, estos métodos no operan adecuadamente cuando se tienen cultivos mixtos, es decir, la presencia de dos o más microorganismos en el medio de fermentación.

\section{Concentración de etanol}

En general, los métodos de medición de esta variable pueden ser divididos dentro de tres categorías: métodos cromatográficos, métodos enzimáticos y métodos ópticos. La cromatografía es la más precisa y sensible, pero es la más costosa; requiere de un pretratamiento en la muestra y consume mucho tiempo de operación. El método enzimático emplea dos enzimas (alcohol oxidasa y alcohol deshidrogenasa) y proporciona sensores altamente selectivos y precisos. Sin embargo, estas enzimas son propensas a la desnaturalización rápida, especialmente cuando se exponen a alta temperatura, presión o $\mathrm{pH}$ extremos.

Los sensores ópticos son menos costosos, de fácil fabricación y están diseñados para el monitoreo en línea y en tiempo real. Sin embargo, su diseño es de baja robustez en su operación, poseen sensibilidad cruzada con el $\mathrm{pH}$ y una baja especificidad (Lam \& Kostov, 2009). Esta variable también puede ser estimada empleando métodos de medición basados en FIA (Flow injection analysis) (Beutel \& Henkel, 2011).

El factor principal en la selección de un método de medición para las diferentes variables de los bioprocesos, es la estabilidad del instrumento, no solo durante los procedimientos de esterilización, sino también por cualquier interacción entre el sensor y los diversos componentes del medio de cultivo que puedan causar interferencias. Otro factor importante es el tiempo de procesamiento (Beutel \& Henkel, 2011).

La ubicación de los instrumentos también afecta la medición. Los sensores son insertados dentro del medio con soportes diseñados especialmente para estas aplicaciones (p. e., sistemas autolavables). En escala industrial, pueden existir problemas de homogeneidad del medio, por lo cual es importante ubicar adecuadamente las sondas de medición o afrontar el problema colocando varias sondas en diferentes lugares (Harms et al., 2002). En lo posible, es mejor ubicar el sensor de tal manera que esté inmerso en el medio continuamente. Aunque existen aplicaciones que no lo permiten, por lo cual es necesario concebir una ubicación en bypass. La medición en bypass es un tipo especial de análisis in situ y algunas veces aplicada, cuando la rata de burbujeo es alta en el tanque reactor. Según la aplicación, los datos obtenidos en bypass pueden diferir de los datos obtenidos directamente del medio, ya que en el tubo de desvío pueden haber fenómenos de no homogeneidad en temperatura, oxígeno disuelto, $\mathrm{pH}$, entre otros (Beutel \& Henkel, 2011).

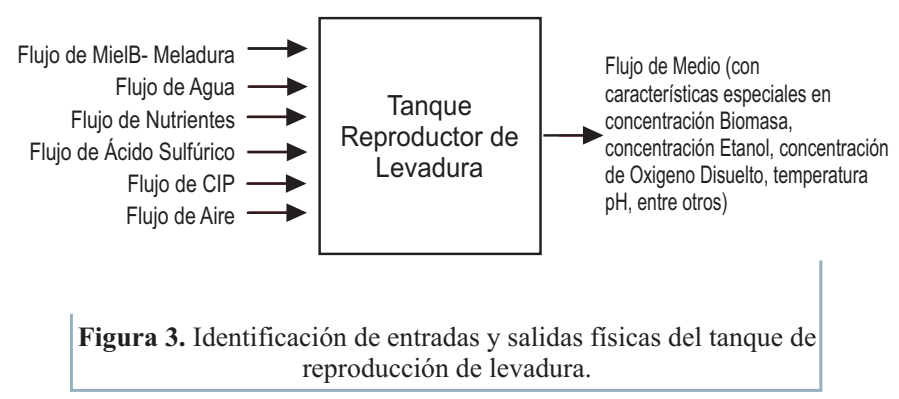




\section{Proceso de Reproducción de Levadura}

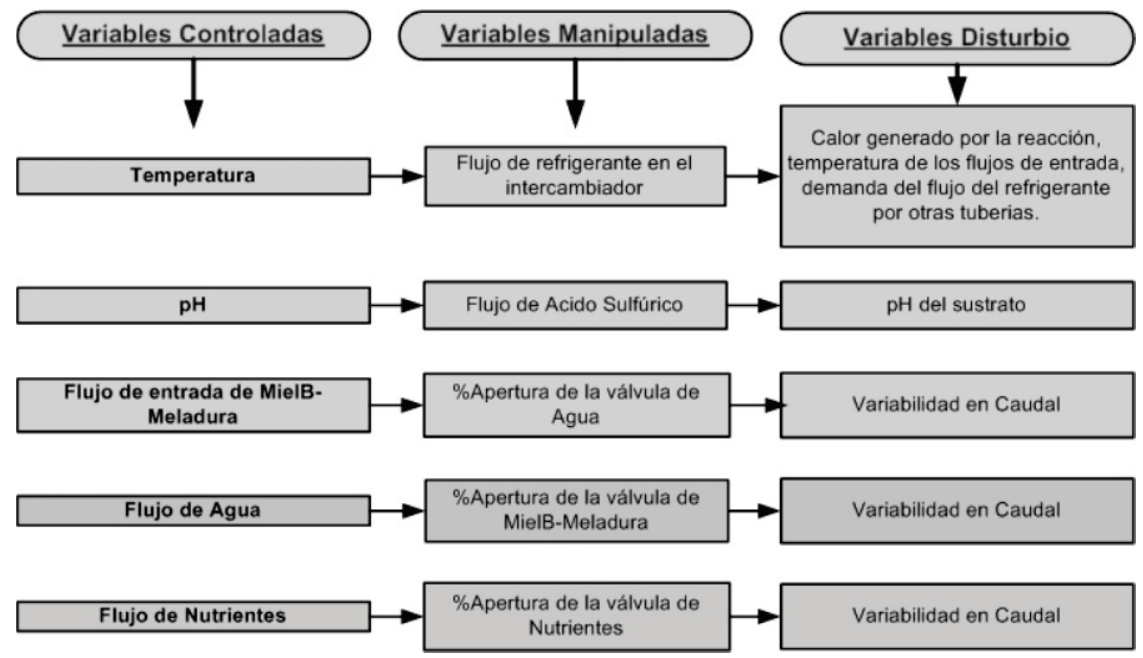

Figura 4. Identificación de las variables del proceso de reproducción de levadura.

\section{PROPUESTA DE AUTOMATIZACIÓN}

\subsection{Identificación de variables para instrumentar}

Con base en el conocimiento del proceso y su operación, se identifican las variables del proceso de reproducción de levadura en continuo. En la Figura 3 se observan las entradas y salidas totales del proceso (las cuales son pautas para establecer instrumentación), y en la Figura 4 se aprecian las variables propias del proceso.

De acuerdo con la insignia citada por Alford (2006), se procede a instrumentar y controlar variables físico-químicas que permitan garantizar un ambiente adecuado para la reproducción de la levadura. Variables críticas del proceso como concentración de etanol, concentración de sustrato y concentración de biomasa, no monitorean ni controlan, porque tienen comportamientos altamente no lineales con modelos poco precisos, y la instrumentación existente para escala industrial aún no es lo suficientemente robusta y confiable.

Además, el mosto del proceso de reproducción de levaduras tiene características particulares que dificultan el monitoreo de la biomasa. Entre esas particularidades se tiene la presencia de otros microorganismos diferentes de la levadura, tales como bacterias ácido-lácticas y bacterias ácido- acéticas que perturbarían significativamente la medición de concentración de biomasa por métodos ópticos, como los existentes en el comercio. Teniendo en cuenta lo anterior, las variables del proceso y parámetros de operación que se instrumentan y regulan para mantener condiciones ambientales y nutricionales adecuadas para la reproducción de la levadura son: temperatura, $\mathrm{pH}$, flujo de MielB-Meladura, flujo de agua, flujo de nutrientes, flujo de salida de mosto y el nivel del medio (volumen del tanque, el cual se regula automáticamente con la regulación de los flujos de entrada y salida del tanque).
Las variables y parámetros que se instrumentaron pero no se controlaron, son: temperatura de reciclo, flujo de aire, concentración de oxígeno disuelto y densidad. La densidad del medio es un indicador del proceso de fermentación, ya que cuando baja a un determinado valor, establecido por el protocolo de operación del proceso, indica que las levaduras están consumiendo el sustrato. Es una medición indirecta de la actividad del sustrato.

\subsection{Requerimientos de instrumentación}

Para el diseño del esquema de automatización se desarrolla un análisis de ingeniería sobre los diferentes aspectos de instrumentación y control del ambiente físico-químico del proceso, el cual contiene dos etapas: ingeniería conceptual e ingeniería básica. Con base en dicho análisis, se establecen los requerimientos de la instrumentación.

En la etapa de ingeniería conceptual, se caracteriza el proceso desde el punto de vista ambiental y de operación, para identificar las necesidades que se tienen y se hace un estudio de involucrados, para definir los beneficios que trae el proyecto sobre cada rol que se desempeña en el proceso. La etapa de ingeniería básica se divide en cuatro secciones, que se revisan a continuación.

5.2.1 Proceso. Se definen aspectos de operación y funcionalidad del sistema, tales como alarmas y enclavamientos, establecidas con base en los rangos de operación dictados por el protocolo de operación del proceso; se determinan los lazos de control con sus respectivos diagramas de lazo, los cuales son establecidos basados en la disposición de conexiones que tiene el DCS de la planta y se diagramaron con la norma ISA 5.4; por último se establece el diagrama PID, el cual contempla toda la instrumentación que requiere el proceso para garantizar una condiciones físicoquímicas adecuadas para la reproducción de levadura. El diagrama PID se observa en la Figura 5. 
5.2.2 Instrumentación. Los instrumentos para medición y actuación, siguiendo los requerimientos del proceso, para las diferentes variables, son:

Elflujo de nutrientes, de mielB-meladura y del medio de salida, son medidos con un instrumento de principio magnético, porque son fluidos particulados (fluidos sucios) y tienen conductividad (requerida por el principio magnético). La medición del flujo de agua de proceso y del fluido refrigerante (agua a $25^{\circ} \mathrm{C}$ ) se hace con una placa de orificio. El instrumento es elaborado en acero inoxidable, que soporta la corrosión. La medición de flujo de aire se hace con un tubo Annubar, este instrumento es adecuado para la medición de gases limpios en tuberías grandes.

La medición de $p H$ del medio se hace con un sensor electroquímico de mínimo mantenimiento con un sistema de autolavado, para evitar las incrustaciones de levadura y partículas del medio que perturbarían la medición. La medición de la densidad del medio se hace con un instrumento cuyo principio se basa en medir la frecuencia de resonancia, y es un instrumento con un índice bajo de mantenimiento. La medición de la concentración de oxígeno disuelto en el medio, se hace con un sensor óptico, basado en el principio de la luminiscencia, adecuado para medir concentraciones bajas de oxígeno disuelto, tal como las que se tienen en los mostos de fermentación. Es un instrumento con un bajo índice de mantenimiento.

Para la medición de la temperatura de medio y del reciclo se emplean RTD, las cuales son apropiadas para procesos industriales como la fermentación.
Tienen la ventaja de ser lineales y de resistir temperaturas altas (hasta los $400{ }^{\circ} \mathrm{C}$ ). La medición del nivel del medio se hace con un sensor tipo capilar de principio de presión diferencial, ya que es un líquido que no tiene cambios de fase. La medición de presencia de espuma se hace con un switch de nivel, el cual se ubica a una determinada altura, de tal manera que cuando se genera abundante espuma, se activa el switch y se informa de la presencia anormal de espuma en el tanque.

Se emplean actuadores neumáticos para contribuir al ahorro de energía eléctrica y también porque se cuenta con suficiente flujo de aire para la realización de esta tarea. Para el control de flujo de nutrientes y MielB-Meladura se emplean válvulas reguladoras tipo bola segmentada (V-ball), ya que son adecuadas para fluidos particulados o con gran porcentaje de sólidos suspendidos.

Para el control del flujo de agua de proceso, medio y refrigerante, se emplean válvulas reguladoras tipo mariposa porque son apropiadas para fluidos a baja presión, fluidos limpios o con bajos porcentajes de sólidos suspendidos y también para uso sobre fluidos corrosivos. Para fluidos como: $C I P$ (en el tanque y en el intercambiador), medio de fermentación a la entrada y salida del intercambiador se emplean válvulas on/off tipo mariposa con confirmación, para manejar cierre o apertura desde el DCS. Para el control del flujo de ácido sulfúrico se emplea una válvula on/off tipo bola en PVC, ya que es adecuada para líquidos viscosos y su material es resistente a la acción del ácido.

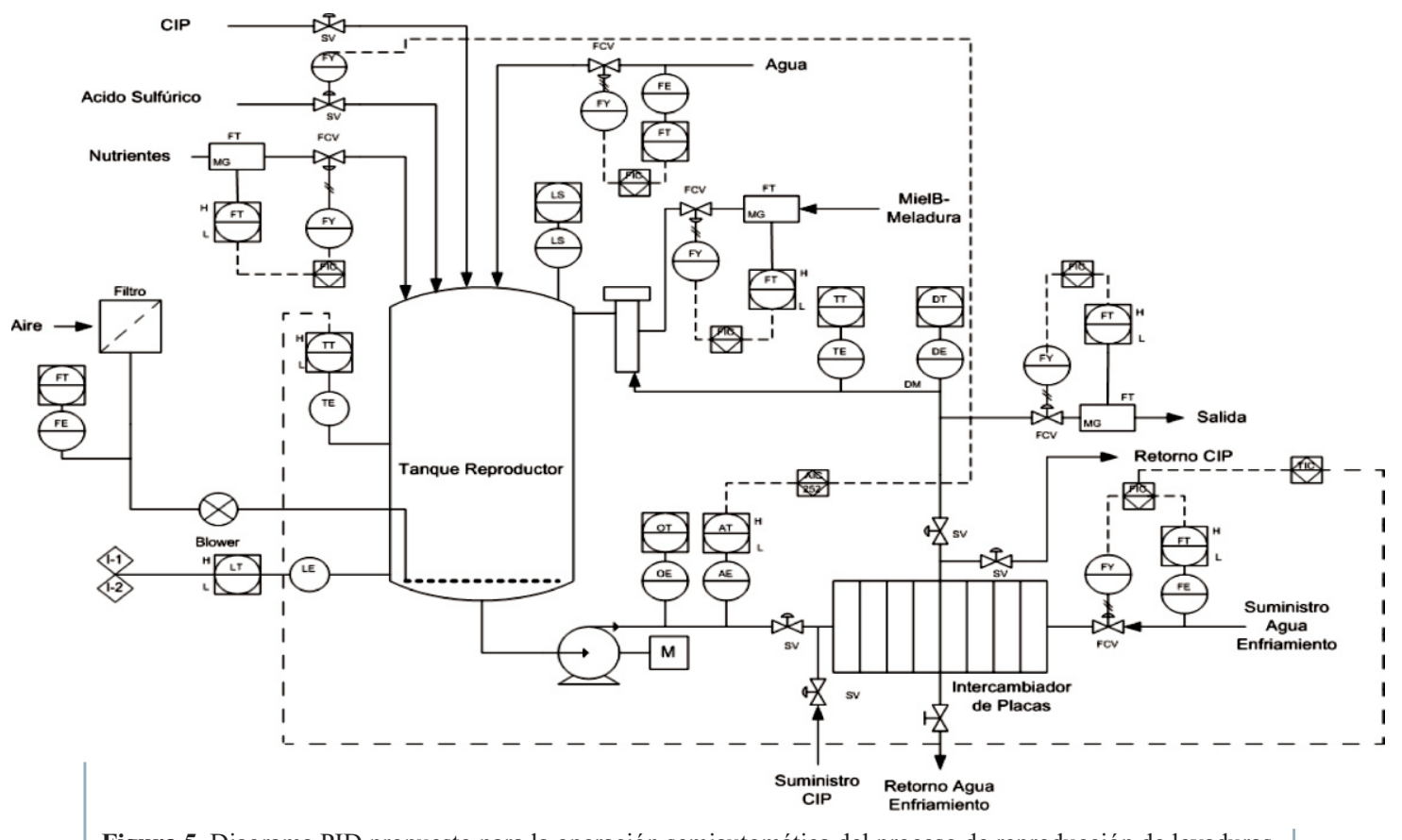

Figura 5. Diagrama PID propuesto para la operación semiautomática del proceso de reproducción de levaduras.

5.2.3 Redes de campo. En la implementación se consideró integrar la solución con el DCS, con el cual cuenta la planta, se determina su arquitectura y la articulación de la propuesta de automatización a la plataforma existente. Además se definieron los PI\&D, las conexiones con el protocolo 4-20mA, la ruta de cableado, los insumos para la conexión de los diferentes instrumentos de campo y el levantamiento del carnet de cableado.
5.2.4 Interfaz humano-máquina. Se define cómo va a ser la HMI del proceso. Esta HMI debe contar con un diseño similar a los existentes en la planta y con las mismas prestaciones. Esto incluye el mismo código de colores y figuras, el mismo esquema de almacenamiento y la misma funcionalidad. 


\subsection{Esquemas de control}

Debido a la naturaleza no lineal y variante en el tiempo del proceso de reproducción de levadura y a la ausencia de instrumentos comerciales confiables, robustos y estables para el monitoreo de variables críticas como la concentración de biomasa, se plantea un esquema de automatización de grado semiautomático para el proceso en general, con controladores regulatorios para las variables flujo y temperatura y un controlador on/off para el $\mathrm{pH}$.

Aunque los procesos de fermentación sostienen un acople entre sus variables, es posible adoptar las variables temperatura, $\mathrm{pH}$, flujos de alimentación y flujo de medio de salida, como sistemas SISO. Ya que se asumen estas variables como condiciones físico-químicas que garantizan un adecuado desempeño en la fermentación que realiza el microorganismo. Para el modelamiento de los comportamientos de estas variables se emplea una técnica de modelado experimental, con base en la metodología de curva de reacción, para encontrar los parámetros y el perfil del modelo.

Los sistemas de flujo están compuestos por la tubería de dosificación, un sensor de flujo y una válvula regulatoria. Para los flujos de nutrientes, MielB-Meladura, agua de proceso y flujo medio de salida, se encuentra que se pueden asociar a comportamientos con dinámicas de primer orden (ver Figura 6), que relacionan el porcentaje de apertura de las válvulas regulatorias con sus respectivos flujos (ver ecuación 6).

$$
\mathrm{G}(\mathrm{s})=\frac{\mathrm{F}(\mathrm{s})}{\mathrm{A}(\mathrm{s})}=\frac{k_{P}}{\tau s+1} m^{3} / \text { hora }-\% \text { Apertura }
$$

Para su adecuado control, se propone un esquema de compensación en serie PI con antiwindup (Figura 7), para evitar errores de estado estable que se reflejen en dosificaciones inadecuadas al proceso. En la Figura 8, la simulación del control propuesto en uno de los sistemas de flujo.
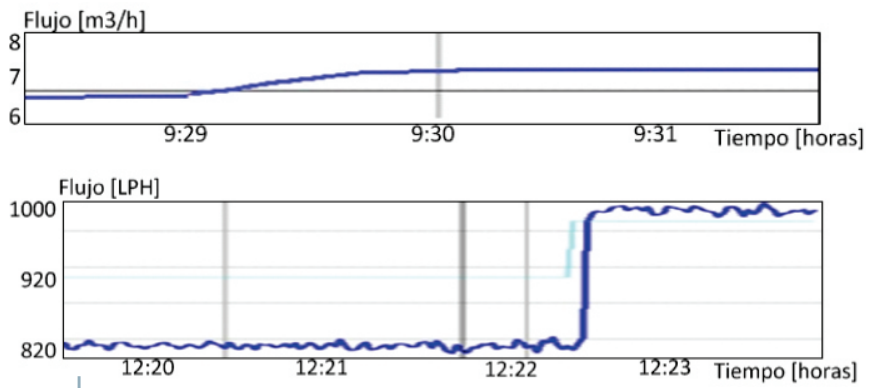

Figura 6. Curvas de reacción del sistema: agua de proceso (curva superior) y MielB-Meladura (curva inferior

El sistema de temperatura está compuesto por un sensor de temperatura ubicado en el tanque y un intercambiador de placas paralelas, por donde circula el medio del tanque y el refrigerante, con una válvula regulatoria que controla el flujo del refrigerante.

La dinámica de este sistema se simplifica partiendo la planta en dos, obteniendo una primera dinámica (ver ecuación 7) que obedece a un sistema de primer orden, el cual relaciona el porcentaje de apertura de la válvula reguladora del flujo refrigerante con el flujo del refrigerante. Una segunda dinámica (ver ecuación 8), que obedece a un sistema de primer orden con tiempo muerto, que relaciona la temperatura del tanque reproductor con el flujo de refrigerante.

$$
\begin{aligned}
& \mathrm{G}_{1}(\mathrm{~s})=\frac{\mathrm{F}(\mathrm{s})}{\mathrm{A}(\mathrm{s})}=\frac{k_{P 1}}{\tau_{1} s+1} m^{3} / \text { hora }-\% \text { Apertura } \\
& \mathrm{G}_{2}(\mathrm{~s})=\frac{\mathrm{T}(\mathrm{s})}{\mathrm{F}(\mathrm{s})}=\frac{k_{P 2}}{\tau_{2} s+1} e^{-t_{m} s} \circ \mathrm{F}-\text { hor } / \mathrm{m}^{3}
\end{aligned}
$$

Para el control adecuado de temperatura, se propone un esquema de compensación en cascada con controladores regulatorios en serie. Donde el controlador maestro obedece a una dinámica PI y el controlador esclavo a un P. La compensación en cascada mejora el desempeño del sistema, siempre y cuando la dinámica interna sea más rápida que la externa, lo cual se cumple claramente en esta aplicación en particular (lazo interno obedece a flujo y lazo externo a temperatura). Otro beneficio de la compensación en cascada es que minimiza el efecto de las perturbaciones que pertenecen al lado de retroalimentación simple, tratando de anticiparse a la variación de la variable por controlar. El esquema de control propuesto se observa en la Figura 9.

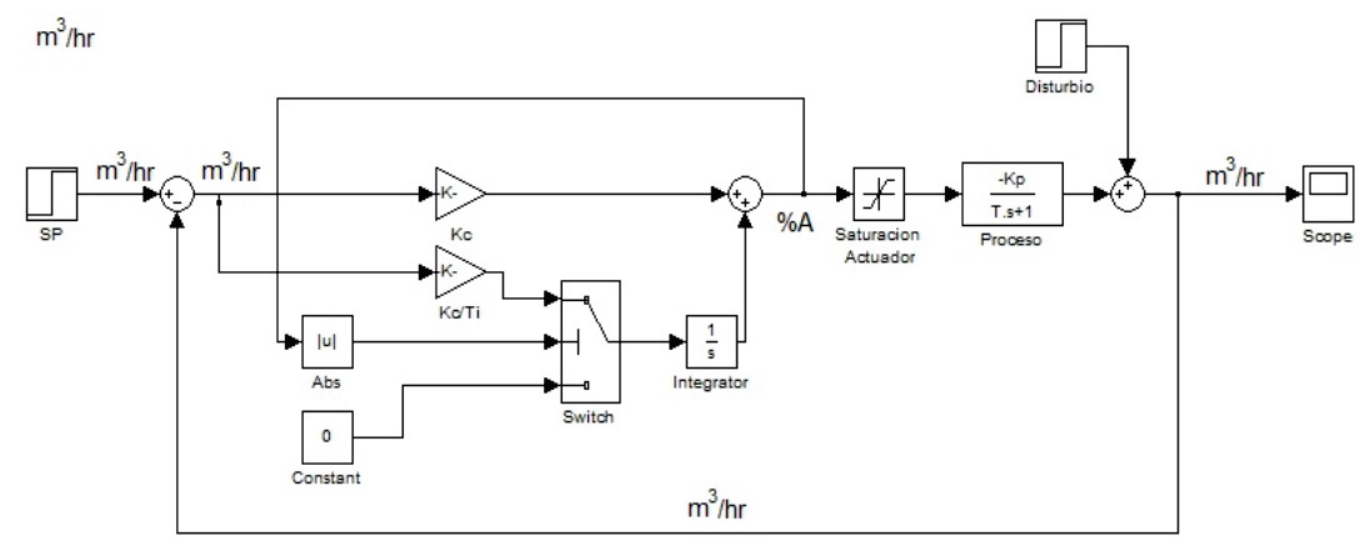

Figura 7. Esquema de control propuesto para regular los flujos de entrada y salida del proceso de reproducción de levaduras. 
El sistema de $\mathrm{pH}$ está compuesto por un sensor de $\mathrm{pH}$ ubicado en el tanque reproductor y una válvula dosificadora on/off de ácido sulfúrico. Se emplea solamente ácido, porque el medio tiene tendencia a volverse básico, debido a la adición continua de miel B, meladura y agua. La dosificación de ácido se hace cuando el $\mathrm{pH}$ está por encima de 5 y durante un lapso máximo de 30 segundos. El protocolo de operación lo establece de esta forma, porque una abundante cantidad de ácido sulfúrico en el proceso de reproducción, puede matar las levaduras o inhibirlas. Además, estas dosificaciones no son tan continuas, debido a que los metabolitos de la fermentación tienden a ser ácidos y los nutrientes (urea y ácido fosfórico) también contribuyen a mantener el $\mathrm{pH}$ por debajo de 5 .

Con base en el manejo del sistema de $\mathrm{pH}$ del tanque reproductor, se propone un esquema de control en compensación en serie, retroalimentado con un controlador on/off.

\subsection{Sintonización de controladores}

Para encontrar los parámetros adecuados de los diferentes controladores, se propone emplear técnicas ampliamente usadas en la industria, como lo son la sintonía de parámetros por medio de tablas de sintonización. Entre los métodos se encuentran: método de síntesis directa, método mínimo error integral ISE, método mínimo error integral IAE y método mínimo error integral ITAE. Igualmente se propone realizarlo con el método de diseño de controladores mediante la asignación de polos. Este método es más efectivo y preciso, pero con más restricciones en su aplicación y consume mayor análisis matemático.

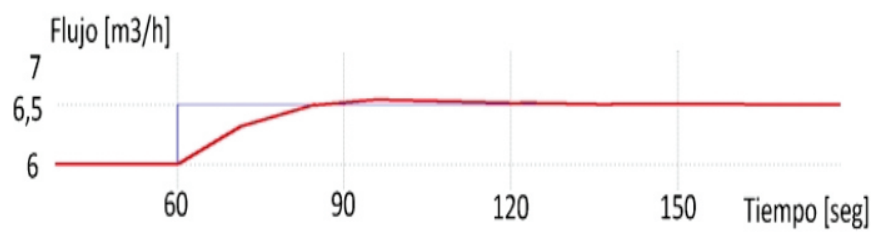

Figura 8. Simulación del esquema de control propuesto para el sistema de flujo de agua del proceso

\section{CONCLUSIONES}

Actualmente es muy difícil realizar un control totalmente automático para el proceso de reproducción de levaduras, porque el proceso es altamente no lineal y variante en el tiempo, no se cuenta con modelos precisos y hay ausencia de instrumentos en línea y en tiempo real para la medición de variables críticas como la concentración de biomasa.

El esquema de automatización propuesto garantiza el cumplimiento de la mayoría de las condiciones ambientales, físicas y químicas necesarias para que la levadura pueda reproducirse, dando cumplimiento a la consigna indicada en Alford (2006).

El esquema semiautomático propuesto, es el primer paso para concebir un esquema totalmente automático para el proceso de reproducción de levaduras.
Los pasos que deben seguirse son la identificación y establecimiento de un modelo preciso, el estudio de estabilidad, controlabilidad y obsevabilidad, la propuesta de un método de estimación de variables y control que se ajuste a los DCS industriales.

La instrumentación comercial para el monitoreo directo de variables críticas del proceso, como la concentración de biomasa, la concentración de producto y la concentración de sustrato para procesos de fermentación, aún no es lo suficientemente robusta, confiable y estable para operar en ambientes industriales.

\section{AGRADECIMIENTOS}

Agradecemos el apoyo financiero de COLCIENCIAS, mediante su programa Jóvenes Investigadores e Innovadores "Virginia Gutiérrez de Pineda"; la colaboración, el apoyo y el valioso interés por la investigación del equipo de operaciones de la planta de destilación del Ingenio Providencia S.A; el apoyo y las orientaciones de los integrantes del "Grupo de Investigación en Control Industrial" (GICI) de la Escuela de Ingeniería Eléctrica y Electrónica, Facultad de Ingeniería de la Universidad del Valle.

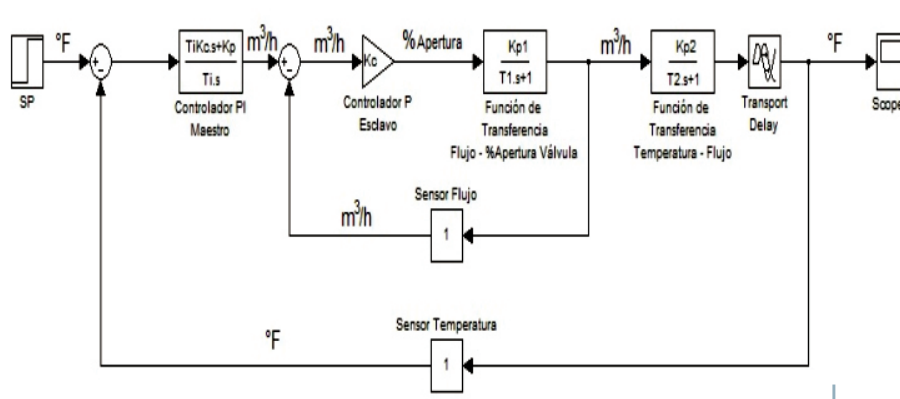

Figura 9. Esquema de control propuesto para regular la temperatura del tanque reproductor de levadura.

\section{REFERENCIAS}

Acevedo, S., Llano, F., Ochoa, J., Parra, J., Calero, L., Caballero, M., Figueroa, M., Marriaga, N. \& Vallejo, R., (2005). Producción de alcohol carburante - Praj. Cenicaña.

Alford, J. S. (2006, Sep.). Bioprocess control: Advances and challenges. Computers \& Chemical Engineering, 30(10-12), 1464-1475.

Ashoori, A. Ghods, H. \& Khaki-sedigh, A. (Dec. 2008). Model Predictive Control of a Nonlinear Fed- Batch Fermentation Process. Control, Automation, Robotics and Vision, 2008. ICARCV 2008. 10th International Conference, p. 17-20.

Atsushi, V. \& Aoyama, V. (1995, Dec.). Internal Model Control Framework Using Neural Networks. Engineering Applications of Artificial Intelligence, 8 (6), 689-70.

Becker, T., Hitzmann, B., Ralf, K. M., Reardon, K. F., Stah,F. 1, \& Ulber, R. (2007). Future Aspects of Bioprocess Monitoring. Adv Biochem Engin/Biotechnol, 249-293.
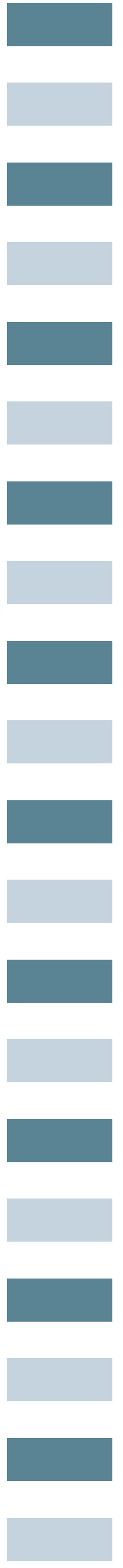
Dochain D. \& Bastin, G. (1990). On-line estimation and adaptive control of bioreactors. USA: Elsevier Science

Dovzan, D. \& Skrjanc, I. (2010). Predictive functional control based on an adaptive fuzzy model of a hybrid semi-batch reactor. Control Engineering Practice, 18, 979-989.

Echeverry, M. R., Álvarez, H. \& Quintero, O. (2003). Control de un biorreactor para fermentación alcohólica en continuo. Bogotá: Universidad Nacional de Colombia.

Harms, P., Kostov, Y. \& Rao, G. (2002). Bioprocess monitoring. Current Opinion in Biotechnology, 124127.

Hernández, C., Escobar, A. \& Galvis, J. (2003, marzo). Control difuso adaptativo aplicados a procesos de fermentación. Rev. Fac. Ing. Univ. Antioquia. (58), 105-113.

Jiang, J., Fan, S., Kong, B., Cai, H., Guo, M. \& Zhuo, V. (2009). Bioprocess Automation Using New Online Sensors. Yeast, 910-914.

Lam, H. \& Kostov, Y. (2010). Optical Instrumentation for Bioprocess Monitoring. Optical Sensor Systems in Biotechnology Advances in Biochemical Engineering / Biotechnology. 116, 125-142.
Lederberg, J. \& Schaechter, M. (2004). The Desk

Encyclopedia of Microbiology. San Diego, CA: Academic Press.

Mantovaneli, I. C. C. \& Filho, R. M. (1992). Hybrid Neural Network Model For Alcoholic. Chemical Engineering, $1-10$.

Meleiro, L., Von Zuben, F. J. \& Filho, R. M. (2009). Constructive learning neural network applied to identification and control of a fuel-ethanol fermentation process. Engineering Applications of Artificial Intelligence, 22( 2), 201-215.

Merchuk, J. C. (1988). Microbiología Industrial. Organización de los Estados Americanos.

Quintero, O., Amicarelli, A. \& Scaglia, G. (2009). Control based on numerical methods and recursive bayesian estimation in a continuous alcoholic fermentation process. Bioresources. 4, 1372-1395.

Thatipamala, S. R. R. \& Hill, G.A. (1993). On-line State and Parameter Estimation and Adaptive Optimization of a Continuous Bioreactor (Ethanol Fermentation ) Using State Equations. American Control Conference. 905909. 